\title{
Experiences of a health coaching self-management program in patients with COPD: a qualitative content analysis
}

This article was published in the following Dove Press journal: International Journal of COPD

Lan Wang ${ }^{1,2}$

Jan Mårtensson'

Yue Zhao ${ }^{2}$

Annette Nygårdh'

'School of Health and Welfare, Jönköping University, Jönköping, Sweden; ${ }^{2}$ Department of Nursing, School of Nursing, Tianjin Medical University, Tianjin, People's Republic of China
Correspondence: Lan Wang School of Nursing, Tianjin Medical University, No 22 Qixiang Road, Heping District, Tianjin 300070, People's Republic of China Tel/fax +862283336910 Email wanglan0423@hotmail.com
Purpose: To describe the experiences of patients with COPD participating in a health coaching self-management program.

Patients and methods: Twenty patients who had participated in a 6-month health coaching self-management program intervention were purposefully selected for a qualitative evaluation of the program using semi-structured interviews. The interviews were analyzed using inductive qualitative content analysis.

Results: Four categories and 13 subcategories emerged describing the participants' experiences of the program. Their experiences were expressed as gaining insight into the importance of knowledge and personal responsibilities in the management of COPD, taking action to maintain a healthy lifestyle, feeling supported by the program, and being hindered by individual and program limitations.

Conclusion: Iterative interactions between patients and health care professionals together with the content of the program are described as important to develop skills to manage COPD. However, in future self-management programs more awareness of individual prerequisites should be considered.

Keywords: chronic obstructive pulmonary disease, health coaching, qualitative content analysis, self-management

\section{Introduction}

COPD has been a leading cause of chronic morbidity and mortality in the world, with a growing economic and social burden ${ }^{1}$ and an estimated global prevalence of $11.7 \%{ }^{2}$ In People's Republic of China, the most recent statistics showed that the pooled prevalence of COPD was nearly 10\% from 1990-2014. ${ }^{3}$ The symptoms of COPD are periodically exacerbated, such as breathlessness, severe cough, and expectoration, accompanied by fatigue, depression, malnutrition, sleep disturbances, and limitations on daily living, which can result in repeated hospitalization and increased mortality. ${ }^{4}$ To prevent the burden on patients and the health care system, raising awareness and knowledge of relevant problems is necessary prerequisites to changing patient behaviors. ${ }^{5}$ Poor knowledge and low awareness of COPD are challenges that lead to serious problems for patients in People's Republic of China. ${ }^{6,7}$ A reason could be that the management of COPD is undertaken by physicians and nurses at general hospitals. Thus, most nurses in primary health care institutions are unfamiliar with COPD guidelines and are therefore not involved in rehabilitation management, follow-up visits, or home care of stable COPD patients after hospitalizations. ${ }^{8}$ 
Self-management for patients with chronic conditions refers to "the individual's ability to manage the symptoms, treatment, physical, and psychosocial consequences and lifestyle changes inherent in living with a chronic condition ...," which has been shown to be a useful strategy to assist patients in taking control of the management of their disease. ${ }^{10}$ Self-management interventions for COPD were highlighted in the latest report of the Global Initiative for Chronic Obstructive Lung Disease. ${ }^{11}$ A recent definition of COPD self-management interventions is: " ... a structured but personalised and often multi-component, with goals of motivating, engaging, and supporting the patients to positively adapt their health behaviours and develop skills to better manage their disease." ${ }^{12}$ A review of the contents of self-management interventions for patients with COPD shows that medication adherence, inhaler technique, smoking cessation, nutritional support, promoting exercise, dyspnea management, recognition of exacerbating symptoms, and psychosocial support often is included. ${ }^{13}$ Self-management programs that support patients with COPD have been shown to decrease hospital admissions, improve health-related quality of life, and increase disease-specific knowledge..$^{14,15}$ However, there are insufficient data to make clear recommendations for effective forms of such programs due to diversity in the duration, structure, and content; ${ }^{15,16}$ low attendance; and high number of dropouts. ${ }^{17,18}$

Health coaching is an interactive person-centered systematic process delivered by health care professionals, in which patients determine their goals to develop accountability in health behaviors and work toward their goals with support from a coach. ${ }^{19}$ Health coaching has emerged as a promising intervention method in recent years and has been effective to improve patients' physiological, behavioral, and psychological conditions..$^{20}$ Health coaching has been used in the follow-up of COPD to motivate patients to be active participants in self-management. ${ }^{20}$ With this in mind, the authors designed and tested a multicenter, 6-month randomized controlled trial of the health coaching self-management program (in press). The intervention was based on a personcentered approach ${ }^{21}$ using health coaching for patients with COPD after hospital discharge.

When evaluating supported self-management programs, a quantitative approach is commonly used. ${ }^{13}$ The only study found using a qualitative evaluation of a self-management program for patients with COPD describe patients' experiences of a minimal (a single 1-hour dialogue and a 20-minute telephone follow-up) home-based psychoeducative intervention to reduce symptoms of anxiety. ${ }^{22}$ The intervention contributed to the patients' ability to self-manage their physical and psychological conditions and was perceived as comprehensible and applicable in the patients' everyday life.

To get a deeper insight into if an intervention program is perceived as meaningful and applicable in a patient's daily life, it is important to use an approach which can provide that information. This information is essential when motivating patients to be active participants and maintaining learned self-management strategies. To our knowledge, there is no study evaluating a comprehensive health coaching self-management program in patients with COPD using a qualitative approach. Furthermore, a health coaching program for patients with COPD has not before been evaluated in People's Republic of China.

Therefore, the aim of this study was to describe the experiences of patients participating in a health coaching selfmanagement program for patients with COPD in People's Republic of China.

\section{Patients and methods Design}

This qualitative study was part of a major multicenter, 6-month randomized controlled trial of a heath coaching self-management program. An inductive qualitative content analysis ${ }^{23}$ was used to describe the intervention as experienced by the participants.

\section{Participants}

Participants $(n=20)$ of those who were allocated to the intervention group $(n=48)$ were purposively selected by criterion sampling ${ }^{24}$ regarding the participants' gender, age, educational level, work, and severity of disease to accomplish a variation of perspectives (Table 1). The inclusion criteria were as follows: 1) forced expiratory volume in 1 second/ forced vital capacity $\leq 70 \%$ diagnosis of COPD according to the Global Initiative for Chronic Obstructive Lung Disease criteria, ${ }^{11}$ and 2) fulfilment of the entire 6-month health coaching program. The exclusion criteria were as follows: 1) acutely exacerbated COPD, and 2) patients who were unable to participate in an interview due to their physical condition or timing issues (Table 1).

\section{Intervention}

The intervention group received a 3-step nurse lead program with a combination of 1 face-to-face coaching (in hospital) and monthly telephone coaching (after discharge) (Figure 1). Before discharge, a face-to-face consultation between the patient and nurse-coach was conducted focusing on patients' 
Table I Sociodemographic and clinical characteristics of the 20 participants

\begin{tabular}{|c|c|c|c|c|c|}
\hline Number & Gender & Age & $\begin{array}{l}\text { Educational } \\
\text { level }^{\mathrm{a}}\end{array}$ & Work $^{\mathrm{b}}$ & $\begin{array}{l}\text { Severity } \\
\text { of disease }\end{array}$ \\
\hline I & Male & 69 & Medium & White collar & GOLD IV \\
\hline 2 & Female & 78 & High & White collar & GOLD III \\
\hline 3 & Male & 55 & Medium & Blue collar & GOLD IV \\
\hline 4 & Female & 62 & High & White collar & GOLD II \\
\hline 5 & Female & 73 & Medium & Blue collar & GOLD IV \\
\hline 6 & Male & 65 & Medium & White collar & GOLD IV \\
\hline 7 & Male & 67 & Medium & Blue collar & GOLD II \\
\hline 8 & Male & 72 & Medium & White collar & GOLD II \\
\hline 9 & Female & 73 & Medium & White collar & GOLD II \\
\hline 10 & Male & 63 & Medium & Blue collar & GOLD II \\
\hline II & Male & 74 & Low & Blue collar & GOLD IV \\
\hline 12 & Male & 75 & Medium & Blue collar & GOLD IV \\
\hline 13 & Female & 75 & Low & Blue collar & GOLD III \\
\hline 14 & Male & 61 & Medium & Blue collar & GOLD II \\
\hline 15 & Female & 72 & Medium & Blue collar & GOLD III \\
\hline 16 & Male & 69 & Medium & White collar & GOLD III \\
\hline 17 & Male & 71 & High & White collar & GOLD III \\
\hline 18 & Male & 69 & Low & Blue collar & GOLD IV \\
\hline 19 & Female & 78 & Low & White collar & GOLD II \\
\hline 20 & Female & 52 & Medium & White collar & GOLD IV \\
\hline
\end{tabular}

Notes: aLow: elementary school or low vocational education; medium: secondary school or intermediate vocational education; high: higher vocational education or university. 'Blue collar: manual worker; white collar: clerical worker. GOLD II: FEV / FVC $<70 \%, 50 \% \leq \mathrm{FEV}_{1}<80 \%$ predicted. GOLD III: $\mathrm{FEV}_{\text {, }} / \mathrm{FVC}<70 \%, 30 \% \leq$ $\mathrm{FEV}_{1}<50 \%$ predicted. GOLD IV: $\mathrm{FEV}, / \mathrm{FVC}<70 \%, \mathrm{FEV}_{1}<30 \%$ predicted.

Abbreviations: $F E V / F V C$, forced expiratory volume in I second/forced vital capacity; GOLD, Global Initiative for Chronic Obstructive Lung Disease.

with interviewing experience using a semi-structured interview guide. The interviews lasted 25-71 minutes and started with an opening question: "Could you describe your experiences in the program you participated in?" The disease knowledge, their social support, self-management level, as well as their beliefs, values, and health goals. With this as a basis, the patient and nurse-coach discussed the plan and reached an agreement involving shared responsibilities to improve the patient's health status. Information about COPD and the patient's plans were summarized in a self-management booklet regarding COPD and a health diary to record their health behaviors, including adherence with medication, smoking status, daily breathing exercise, and other types of daily exercise. Patients were also encouraged to use a pedometer delivered by the coach to monitor their physical activity by step counts and to increase the steps gradually every week.

After discharge, monthly telephone coaching was performed to support and motivate the patient implementing the plan, evaluate improvements in the program, adapt the contents of the program, and make changes according to the patient's symptoms and individual needs. The nurse-coach also provided further guidance by telephone coaching when needed, eg, deteriorations of the condition, difficulty mastering the disease, and adherence.

\section{Data collection}

The interviews were conducted from May 2016 to October 2016. Two pilot interviews were performed by the first author to test the interview guide. One question on the influence on family or friends was added to the interview guide, and the pilot interviews were considered appropriate to be included in the analysis. The other participants were interviewed at their homes or at an outpatient clinic by 2 nurses

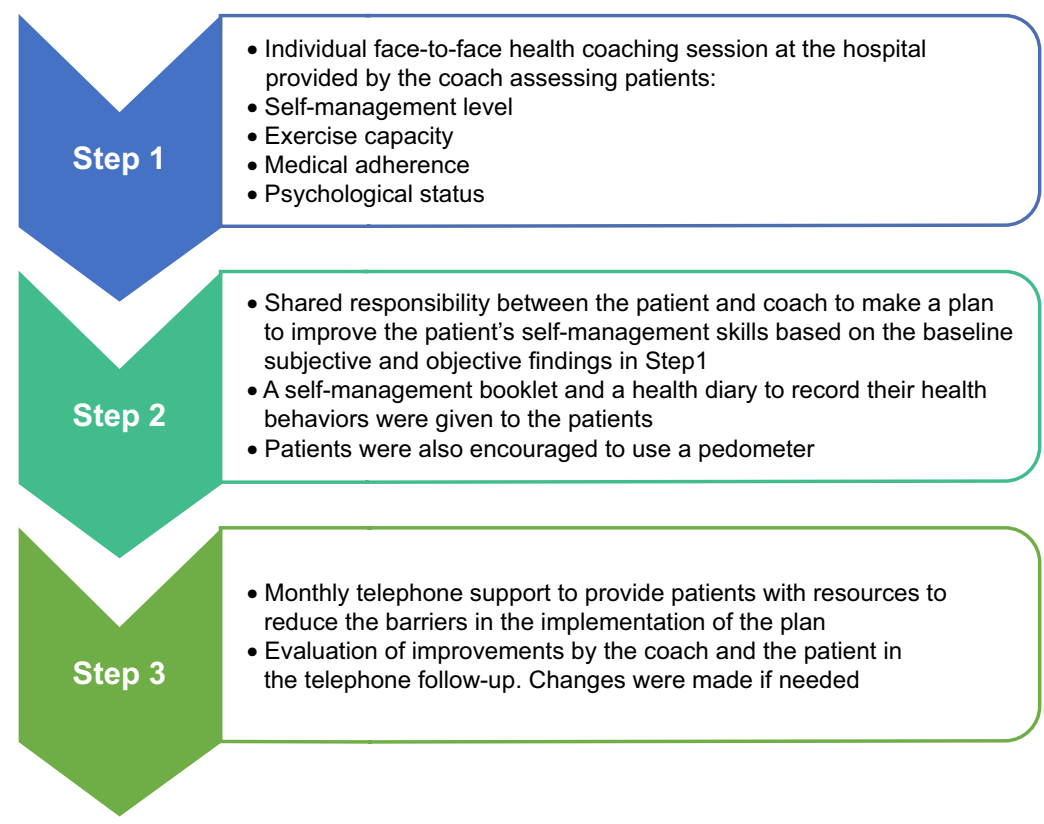

Figure I The content of the intervention of a health coaching self-management program in patients with COPD. 
participants were encouraged to describe their experiences in the program, to reflect on their involvement in the process, and to identify the facilitating factors or barriers to their participation in the program. Different follow-up questions were asked depending on the answer to this question. At the end of the interview, the participants were encouraged to propose some suggestions to improve the program. The interviews were taped with a digital voice recorder and transcribed verbatim.

\section{Data analysis}

The interviewer reviewed the transcripts by listening to the tape recording to ensure its accuracy. The transcripts were analyzed using inductive qualitative content analysis. This means that data move from the specific to the general by open coding, creating categories, and abstraction. ${ }^{23}$ The first 3 interviews were translated into English to allow a thorough discussion among the authors about the emerging meaning units. Afterward, only the meaning units were translated from the other 17 interviews. The data analysis process included open coding, creating categories, and abstraction. ${ }^{23}$ In open coding, the written text was reread several times, and a general sense of the participants' experiences was recorded in the transcript margins as notes or headings. After open coding, the notes and headings were collected from the margins and transferred to a coding sheet where similar notes and headings were grouped together to generate higher order headings. Similar higher order headings were then collected on a separate sheet for analysis, and subcategories were identified. Through abstraction, similar experiences in the subcategories were grouped on a separate sheet and categories were generated. The data analysis was mainly conducted by the first author (LW), and the emerging codes, sub categories, and categories were critically discussed and compared within the research group until agreement was reached. All the authors have extensive experience as a nurse, 2 of the authors (LW and YZ) are familiar with the research field, and the other 2 (JM and AN) have extensive experience using qualitative research methods.

\section{Ethical considerations}

This study was approved by the local ethics committee of Tianjin Medical University, People's Republic of China (TMUEC201400201), and conducted in accordance with the Declaration of Helsinki. ${ }^{25}$ All of the participants were informed of the purpose and procedures of the study. Written informed consent was obtained and confirmed from all participates before data collection. Assurance of confidentiality was given by coding the participants' interviews with numbers at the transcription stage, and ensuring no individual is identifiable in the result. Participation in the study was voluntary, and participants retained the right to withdraw from the study at any time without reason or consequences.

\section{Results}

Four categories and 13 subcategories emerged describing the participants' experiences of participating in the intervention. The participants' experiences were expressed as gaining insight, taking action, feeling supported, and being hindered (Table 2).

\section{Gaining insight}

The category "Gaining insight" describes the participants' experiences of how the program made them more aware of the importance of knowledge of the disease and their own responsibilities. They did not have a comprehensive perception about COPD before attending the program and described how the program helped them learn how to manage the disease, specifically in regard to their knowledge of prevention, rehabilitation, and medical treatment.

\section{Awareness of causes and prognosis}

The participants described how the program helped them become aware of the trajectory of the disease, which they were not aware of before. They learned about the different causes and their impact on the development of COPD. The participants also expressed how the program helped them be aware of the severity and prognosis of the disease.

Table 2 Overview of the subcategories and categories describing experiences of attending a health coaching self-management program for patients with COPD

\begin{tabular}{ll}
\hline Subcategories & Categories \\
\hline Awareness of causes and prognosis & Gaining insight \\
Awareness of COPD management & \\
Awareness of personal responsibility & \\
Seeking information & Taking action \\
Remaining healthy & \\
Enabling activities & \\
Coaching other people & \\
Being reminded and encouraged & Feeling supported \\
Facilitative access & \\
Enlightened family & \\
Limitations in individual prerequisites & Being hindered \\
Limitations in surroundings & \\
Limitations in program contents & \\
\hline
\end{tabular}


I have read the booklet on COPD. I understand that the disease has a relationship to air pollution and haze. I have also been told that it is impossible to be completely recovered because COPD is a chronic disease. (Participant 3)

\section{Awareness of COPD management}

The program helped the participants be aware of the importance of avoiding risk factors for COPD and the benefits of respiratory training techniques and physical activity. They expressed that they became aware of detrimental behaviors, such as unhealthy drinking and eating habits. When they started to attend the program, they reported increased understanding of rehabilitation, such as respiratory training and other tools to guide physical activity. The participants also described their awareness of COPD medications and their side effects. The telephone coaching strengthened their adherence in taking long-term prescriptions and helped them overcome the fear of drug dependence:

I think the coach gave me a lot of good suggestions about disease recovery and treatment, which let me understand how to prevent, how to treat, how to maintain my health. This is my feeling. (Participant 11)

\section{Awareness of personal responsibility}

The participants expressed their awareness of personal responsibility in the management of COPD as depending on themselves but also as a mutual responsibility between health care professionals and patients. The coaches' supervision and concern motivated them to take ownership of their health and increased their awareness of how to be alert to symptoms and the trajectory of COPD.

With the coaching, I know that I should control my disease by myself. I should do breathing exercises, take medicine regularly by myself. I should control my diet, and irritable food cannot be eaten, and I should remember to rest. (Participant 20)

\section{Taking action}

The category "Taking action" describes the participants' experiences of how the program increased their motivation to engage in different physical activities that result in decreased frequencies of exacerbated episodes of COPD and a healthier lifestyle. They also said that they began to actively seek more information regarding the disease from different resources, avoid risk factors and unhealthy behaviors, engage in more activities to manage their symptoms, and coach other patients with COPD in managing the disease.

\section{Seeking information}

The participants reported that the program helped them seek information in order to acquire mastery in understanding their disease. They were actively seeking information regarding COPD from more media sources, such as the Internet, magazines, lectures, television, and newspapers. The participants also emphasized that they did not previously pay attention to useful information on COPD and that the program helped them to realize the importance of acquiring more information on COPD to better cope with their life situation.

I always read the newspaper now. When some health-related information is described in the newspaper, I always cut it out and keep it. I just read it before, but now I pay more attention to it and keep it. (Participant 15)

\section{Remaining healthy}

The program helped the participants stay healthy by avoiding risk factors in their lifestyle habits, such as an unhealthy diet, high cooking fumes, active or passive smoking, and physical inactivity to avoid acute exacerbation of COPD. They said that the program coached them to use different approaches to maintain healthy behaviors, such as wearing a mask and keeping warm when going outside in bad or cold weather.

I ate a lot in the past. Eating without restraint, following my inclinations, drinking and smoking. But now I don't do that. I only eat 3 times every day, and not more even when I'm very hungry. My life habits have changed a lot and I feel comfortable when changing them. (Participant 1)

\section{Enabling activities}

The participants reported that the program enabled them to perform different activities, such as walking every day, attending cultural performances, visiting friends' houses, travelling to other cities, and doing housework. They learned techniques and approaches on how to manage taking their medicine and engaging in activities in a way that reduced symptoms. They also emphasized that respiratory training allowed them to breathe comfortably.

I did abdominal breathing when I felt uncomfortable breathing when climbing the mountain. The breathing then became calm and normal. (Participant 4)

\section{Coaching other people}

Relatives and friends who suffered from the same disease were often coached by the participant on how to improve their physical condition following the guidance of the program. They emphasized that they shared their experiences and 
feelings with people around them and encouraged them to try respiratory training techniques, appropriate approaches to physical activities, good strategies for controlling one's temper, and techniques for smoking cessation.

One of my comrades-in-arms, he was better than before after I coached him about how to do respiratory training and keep a positive attitude. (Participant 1)

\section{Feeling supported}

The category "Feeling supported" describes participants" experiences when they encountered support in various parts of the program, such as telephone coaching, pedometer use, and information booklets. They felt that the program was a convenient way to access responsible coaches. The participants said that the coach's contact strengthened their confidence in coping with dyspnea and in developing self-management skills. The program also facilitated their families' understanding of the disease and how to support the patient.

\section{Being reminded and encouraged}

There were several reminders in the program such as active telephone coaching, pedometer use, and booklet recording that helped the participants maintain pulmonary rehabilitation using respiratory training, physical activities, and medication. The participants also said that the coaches' encouragement improved their emotional well-being. They expressed that the coach's kindness exceeded their expectations, and they felt more optimistic and powerful. These reminders and encouragement also helped them to recognize that they should play an active role in managing and improving their own health.

Coach always contacted me and encouraged me whether it was to exercise, practise respiratory exercises, or take medicine regularly ... reminded me to increase or decrease what I'm wearing and not go outside if the weather is bad. (Participant 7)

\section{Facilitated access}

The participants reported that monthly telephone coaching facilitated access to health care professionals. They said that gaining access to health care professionals after hospital discharge had previously been difficult. The telephone coaching facilitated their understanding and performance of breathing techniques, and they emphasized the importance of the coaches always being available. The telephone coaching increased the participants' opportunities to contact health care professionals when their symptoms worsened or when they were unsure about how to handle their symptoms.

Telephone coaching. Timely contact. I can ask coach when I cannot understand. These aspects will give me a lot of benefits. It is really very good ... for example, how to take medicine, how to do rehabilitation, I can consult with you. (Participant 14)

\section{Enlightened family}

The program enlightened the participants' families who were their main caregivers. The participants said that by taking part in the program, their families learned how to perform respiratory training and prevent cold. The participants also emphasized that their families helped the coaches supervise their actions following the coaches' training and plans.

My family has learned a lot after coach coached me. My daughter didn't know that COPD was so serious. But after you coached me, she learned how to prevent it. (Participant 18)

\section{Being hindered}

The category "Being hindered" describes the participants' experiences with their individual factors, surroundings, and family context that made it difficult for them to follow the program. They also described some shortcomings of the program. The content did not match their needs, and the participants had suggestions on how to improve it.

\section{Limitations in individual prerequisites}

Limitations in individual factors refer to some participants' low literacy, physical degeneration, and other symptoms of disease. They said that they could not remember or understand the contents of the booklet because they were too old. It could also be difficult to read the booklet and record in the diary due to illiteracy, apathy, or fatigue. Some participants also suffered from a variety of diseases that restricted their ability to continue respiratory training for a long time.

I read the booklet sometimes, but I can only understand the pictures because I cannot read the words. I can only understand the picture of the breathing exercise; the others I did not understand. (Participant 18)

\section{Limitations in surroundings}

It was difficult for some of the participants to follow the prescribed program due to their life situations, including needing to do housework or care for their parents, and this hindered them sustaining their rehabilitation efforts. Some participants 
also complained that the medical insurance system inhibited them from taking medicine every day because they had no extra financial resources to afford the expensive drugs.

Sometimes I cannot continue to take medicine, one reason is my medical insurance. I have no money to buy the drug. (Participant 14)

\section{Limitations in program contents}

The participants said that the program had insufficient content and offered some suggestions. They thought that there was too little face-to-face coaching and that the contents of the booklet were inadequate and inflexible. They also reported that it was inconvenient to use the pedometer in the summer, boring to perform respiratory training, and monotonous to record a diary and only walk for exercise. The participants proposed organizing a group where the patients could meet to share their experiences and suggested separating the booklet into 2 parts, one part providing information and the other as a diary.

I record the diary at the beginning after discharge. However,

I only kept the diary for 1 month, and then I didn't want to

do it. It was a little tedious. (Participant 12)

\section{Discussion}

This is the first study to describe the experiences of patients who participated in a health coaching self-management program for patients with COPD in People's Republic of China. The participants increased their insights about their disease and personal accountability. Understanding the progression of the disease helped them change their attitudes toward self-management and comprehend the principles of breathing training and physical activity. Similar perceived benefits have been described in other studies of COPD patients. ${ }^{26,27}$ The COPD patients' knowledge about their disease has been reported to be poor in several aspects, specifically regarding the causes of COPD, the consequences of uncoordinated therapy, and the management of exacerbation. ${ }^{28}$ In a study from People's Republic of China, only 16\% knew how to perform breathing techniques, 50\% had knowledge about regular treatments, and 36\% understood the etiology of COPD. ${ }^{6}$ Increasing awareness by developing and providing an educational program appropriate for the culture has been shown to help COPD patients. ${ }^{29}$

In the current study, the program increased the patients' motivation to play an active role in the self-management process. They were engaged in various healthy activities, such as seeking information on COPD actively and persevering with rehabilitation. In addition, the participants changed their negative behaviors to achieve disease stability. Face-to-face coaching immediately following discharge and a series of telephone coaching sessions has been shown to increase adherence motivation. ${ }^{20}$ Motivation was viewed as a vital factor in the current intervention, which was consistent with several previous studies on exercise and rehabilitation in chronic disease..$^{30}$ Motivation for physical activity has been shown to be positively associated with self-management in patients with COPD. ${ }^{31}$ However, when face-to-face intervention at the hospital ends, the patients sometimes are unable to receive feedback from health professionals due to barriers such as travel time or transportation issues, ${ }^{32}$ and their motivation to continue living a healthy lifestyle through diet and exercise decreases. ${ }^{33}$ Therefore, a health care self-management program including 1 face-to-face coaching before discharge and monthly telephone coaching after discharge seems to be an adequate approach to motivate healthy behaviors.

The participants felt supported by the program, particularly using the pedometer and receiving telephone support from the coach. The pedometer was an incentive to support exercise in the current program because the patients could measure their step counts and encourage themselves to gradually increase the amount of activity. A previous study found that low-intensity physical activity can reduce the risk of COPD hospitalizations, ${ }^{34}$ and pedometers have been shown to effectively improve physical activity levels and reduce exacerbations in patients with COPD. ${ }^{35}$ The patients in the current study also said that telephone support provided access to timely and appropriate care, communication, and coordination between themselves and their health care providers. Telephone support has been increasingly used in recent years in health care ${ }^{36}$ to facilitate patients' access to their health care providers and is a cost-effective approach to support behavioral health changes for patients with diabetes. ${ }^{37,38}$ Patients with COPD often experience insufficient support from their health care providers because too little time is allocated in the outpatient clinic, which leads to a lack of access to resources to help them cope with day-to-day problems. ${ }^{39}$ Telephone support for long-term conditions has potential benefits for self-efficacy, health behavior, and health status because it can gradually improve patients' skills and tailor support to the individual patient's needs. ${ }^{26,40}$

Although patients gained more awareness of their disease and their own responsibilities in managing it, accomplishing changes in self-management and lifestyle are not feasible for everyone. Previous research on health coaching programs 
showed that the level of patient participation in health care decisions can vary greatly. ${ }^{41}$ In the current study, the participants described individual limitations to improving their health by themselves due to age, literacy, symptoms, and their life situation regarding workloads and economic issues. This is in line with previous research on factors influencing self-management in diabetes care in People's Republic of $\mathrm{China}^{42}$ and empowerment in patients with COPD. ${ }^{43}$ Some environmental barriers cannot be solved by health care providers in People's Republic of China, such as insufficient income. Therefore, the involvement of interdisciplinary teams in health coaching programs should be considered in order to improve person-centered care. ${ }^{44}$

\section{Methodology considerations}

This study adopted the criteria for qualitative research introduced by Lincoln and Guba. ${ }^{45}$ To strengthen the credibility, scientific and rigorous processing of the data was performed. The dependability of the study was enhanced by using a semistructured interview guide which directed the interview. The first author was familiar with the context of transcription, and continuous discussion was carried out among all the authors during the analysis process to reach consensus, which further strengthened the dependability. To identify and select a variety of information-rich cases who had participated in the intervention, a purposeful criterion sampling was used, which enhanced the credibility and transferability of the findings.

\section{Limitations}

However, this study also has several limitations. First, the initial interviews were transcribed and then translated from Chinese to English, which is a second language for all of the authors. However, all of the authors have experience in chronic disease management and discussed the first 3 interviews to gain a common understanding of these patients' expressed experiences and how to select the meaning units. The analysis was performed in English, and in the discussions between the authors regarding codes, subcategories, and categories, the first author was able to refer back and forth to the transcribed text. Second, some of the interviews were performed at home with the patients' families present, which might have influenced the patients' commentary. However, the families were told before the interviews not to interrupt or attend the dialogue. Third, the coach, who had developed and performed the randomized controlled trial conducted the first 2 interviews in order to test the interview guide, which may have led to the Hawthorne effect, referring to the interviewer's subjectivity influencing the interviews. ${ }^{46}$ However, the remaining 18 interviews were conducted by 2 other nurses.

\section{Conclusion}

This is the first study describing the experiences of patients with COPD who participated in a health coaching selfmanagement program in People's Republic of China. Iterative interactions between patients and health care professionals who are competent in delivering self-management interventions with the goals of motivating, engaging, and supporting the patients are needed to develop skills to better manage COPD. Literacy-sensitive approaches are important to enhance comprehensibility and thereby enable self-management abilities for a larger group of patients with COPD. In future self-management programs, more awareness of individual prerequisites should be considered.

\section{Acknowledgment}

This work was supported by grants from the Tianjin Science and Technology Development Strategy Research Project (Grand No. 17ZLZXZF00480) to Lan Wang. The researchers are grateful to Yan-xia Tao and Xiao-yan Dong for assisting in data collection. The authors would like to express thanks to all the participants who kindly took part in this study.

\section{Disclosure}

The authors report no conflicts of interest in this work.

\section{References}

1. Lozano R, Naghavi M, Foreman K. Global and regional mortality from 235 causes of death for 20 age groups in 1990 and 2010: a systematic analysis for the Global Burden of Disease Study 2010. Lancet. 2012;380(9859):2095-2128.

2. Adeloye D, Chua S, Lee C, et al. Global and regional estimates of COPD prevalence: systematic review and meta-analysis. J Glob Health. 2015;5(2):020415.

3. Bao H, Fang L, Wang L. [Prevalence of chronic obstructive pulmonary disease among community population aged $\geq 40$ in China: a metaanalysis on studies published between 1990 and 2014]. Zhonghua Liu Xing Bing Xue Za Zhi. 2016;37(1):119-124. Chinese.

4. Garcia-Aymerich J, Serra Pons I, Mannino DM, Maas AK, Miller DP, Davis KJ. Lung function impairment, COPD hospitalisations and subsequent mortality. Thorax. 2011;66(7):585-590.

5. Neuner-Jehle S, Schmid M, Grüninger U. The "Health Coaching" programme: a new patient-centred and visually supported approach for health behaviour change in primary care. BMC Fam Pract. 2013;14:100-108.

6. Yang CC. A survey of disease recognition and nursing service needs of chronic obstructive pulmonary disease patients in Zhabei community of Shanghai city. Chin Nurs Res. 2009;23:395-396.

7. Chen YH, Yao WZ, Kang J, et al. [Attitudes and actions of chronic obstructive pulmonary disease patients on treatment: a national multicenter investigative study]. Zhonghua Jie He He Hu Xi Za Zhi. 2010; 33(10):750-753. Chinese. 
8. Cao MJ, Guo XL, Yu H, Chen LY, McDonald TA. Chinese communitydwelling elders' needs: promoting ageing in place. Int Nurs Rev. 2014; 61(3):327-335.

9. Barlow J, Wright C, Sheasby J, Turner A, Hainsworth J. Selfmanagement approaches for people with chronic conditions: a review. Patient Educ Couns. 2002;48(2):177-187.

10. Bucknall C, Miller G, Lloyd S, et al. Glasgow supported selfmanagement trial (GsuST) for patients with moderate to severe COPD: randomised controlled trial. BMJ. 2012;344:e1060.

11. From the Global Strategy for the Diagnosis, Management and Prevention of COPD, Global Initiative for Chronic Obstructive Lung Disease (GOLD). Available from: http://goldcopd.org/gold-2017-global-strategydiagnosis-management-prevention-copd/. Accessed May 30, 2017.

12. Effing TW, Vercoulen JH, Bourbeau J, et al. Definition of a COPD self-management intervention: International Expert Group consensus. Eur Respir J. 2016;48(1):46-54.

13. Majothi S, Jolly K, Heneghan NR, et al. Supported self-management for patients with COPD who have recently been discharged from hospital: a systematic review and meta-analysis. Int J Chron Obstruct Pulmon Dis. 2015;10:853-867.

14. Wang T, Tan JY, Xiao LD, Deng R. Effectiveness of disease-specific self-management education on health outcomes in patients with chronic obstructive pulmonary disease: an updated systematic review and metaanalysis. Patient Educ Couns. 2017;100(8):1432-1446.

15. Zwerink M, Brusse-Keizer M, van der Vaik PD, et al. Self-management for patients with chronic obstructive pulmonary disease. Cochrane Database Syst Rev. 2014;(3):1-118.

16. Keating A, Lee A, Holland AE. What prevents people with chronic obstructive pulmonary disease attending pulmonary rehabilitation? A systematic review. Chron Respir Dis. 2011;8(2):88-89.

17. Taylor SJ, Sohanpal R, Bremner S, et al. Self-management support for moderate-to-severe chronic obstructive pulmonary disease: a pilot randomized controlled trial. Brit J Gen Pract. 2012;62(603): e687-e695.

18. Vercoulen JH. A simple method to enable patient-tailored treatment and to motivate the patient to change behaviour. Chron Respir Dis. 2012;9(4):259-268.

19. Wolever RQ, Simmons LA, Sforzo GA, et al. A systematic review of the literature on health and wellness coaching: defining a key behavioral intervention in healthcare. Glob Adv Health Med. 2013;2(4):38-57.

20. Kivelä K, Elo S, Kyngäs H, Kääriäinen $M$. The effects of health coaching on adult patients with chronic diseases: a systematic review. Patient Educ Couns. 2014;97(2):147-157.

21. Ekman I, Swedberg K, Traft C. Person-centered care-ready for prime time. Eur J Cardiovasc Nurs. 2011;10(4):248-251.

22. Bove DG, Midtgaard J, Kaldan G, Overgaard D, Lomborg K. Homebased COPD psychoeducation: a qualitative study of the patients' experiences. J Psychosom Res. 2017;98:71-77.

23. Elo S, Kyngäs $\mathrm{H}$. The qualitative content analysis process. $J$ Adv Nurs. 2008;62(1):107-115.

24. Palinkas LA, Horwitz SM, Green CA, Wisdom JP, Duan N, Hoagwood K. Purposeful sampling for qualitative data collection and analysis in mixed method implementation research. Adm Policy Ment Health 2015;42(5):533-544.

25. 18th WMA General Assembly. WMA Declaration of Helsinki-Ethical Principle for Medical Research Involving Human Subject. FerneyVoltaire, France: World Medical Association; 1964.

26. Walters JA, Cameron-Tucker H, Courtney-Pratt H, et al. Supporting health behaviour change in chronic obstructive pulmonary disease with telephone health-mentoring: insights from a qualitative study. $B M C$ Fam Pract. 2012;13:55.

27. Liddy C, Johnston S, Irving H, Nash K, Ward N. Improving awareness, accountability, and access through health coaching: qualitative study of patients' perspectives. Can Fam Physician. 2015;61(3):158-164.
28. Hernandez P, Balter M, Bourbeau J, Hodder R. Living with chronic obstructive pulmonary disease: a survey of patients' knowledge and attitudes. Respir Med. 2009;103(7):1004-1012.

29. Grouse L, Nonikov D. The global battle to improve patients' health outcomes: COPD: awareness, activities, and progress. $J$ Thorac Dis. 2014;6(2):161-168.

30. Farholm A, Sørensen M. Motivation for physical activity and exercise in severe mental illness: a systematic review of intervention studies. Int J Ment Health Nurs. 2016;25(3):194-205.

31. Wang L, Nygårdh A, Zhao Y, Mårtensson J. Self-management among patients with chronic obstructive pulmonary disease in China and its association with sociodemographic and clinical variables. Appl Nurs Res. 2016;32:61-66.

32. Jones LW, Guill B, Keir ST, et al. Exercise interest and preferences among patients diagnosed with primary brain cancer. Support Care Cancer. 2007;15(1):47-55.

33. Daley AJ, Crank H, Saxton JM, Mutrie N, Coleman R, Roalfe A. Randomized trial of exercise therapy in women treated for breast cancer. J Clin Oncol. 2007;25(13):1713-1721.

34. Donaire-Gonzalez D, Gimeno-Santos E, Balcells E, et al. Benefits of physical activity on COPD hospitalisation depend on intensity. Eur Respir J. 2015;46(5):1281-1289.

35. Mendoza L, Horta P, Espinoza J, et al. Pedometers to enhance physical activity in COPD: a randomised controlled trial. Eur Respir J. 2015 45(2):347-354.

36. Free C, Phillips G, Galli L, et al. The effectiveness of mobile-health technology-based health behaviour change or disease management interventions for health care consumers: a systematic review. PLoS Med. 2013;10(1):1-45.

37. Kennedy A, Rogers A, Chew-Graham C, et al. Implementation of a self-management support approach (WISE) across a health system a process evaluation explaining what did and did not work for organization, clinicians and patients. Implement Sci. 2014;9:129-145.

38. McGloin H, Timmins F, Coates V, Boore J. A case study approach to the examination of a telephone-based health coaching intervention in facilitating behaviour change for adults with type 2 diabetes. $J$ Clin Nurs. 2015;24(9-10):1246-1257.

39. Wodskou PM, Høst D, Godffredsen NS, Frølich A. A qualitative study of integrated care from the perspective of patients with chronic obstructive pulmonary disease and their relatives. BMC Health Serv Res. 2014;14:471-481.

40. Dennis SM, Harris M, Lloyd J, Powell Davies G, Faruqi N, Zwar N. Do people with existing chronic conditions benefit from telephone coaching? A rapid review. Aust Health Rev. 2013;37(3):381-388.

41. Levinson W, Kao A, Kuby A, et al. Not all patients want to participate in decision making. A national study of public preferences. J Gen Intern Med. 2005;20(6):531-535.

42. Luo XP, Liu TT, Yuan XJ, et al. Factors influencing self-management in Chinese adults with type 2 diabetes: a systematic review and metaanalysis. Int J Environ Res Public Health. 2015;12(9):11304-11327.

43. Harmsen CG, Jarbøl DE, Nexøe J, et al. Impact of effectiveness information format on patient choice of therapy and satisfaction with decisions about chronic disease medication: "Influence of intervention Methodologies on Patient Choice of Therapy (IMPACT)" cluster-randonmized trial in general practice. BMC Health Serv Res. 2013;13:76-83.

44. Liu W, Gerdtz M, Manias E. Creating opportunities for interdisciplinary collaboration and patient-centred care: how nurses, doctors, pharmacists and patients use communication strategies when managing medications in an acute hospital setting. J Clin Nurs. 2016;25(19-20):2943-2957.

45. Lincoln YS, Guba EG. Naturalistic Inquiry. Thousand Oaks, CA: Sage Publications; 1985.

46. Paradis E, Sutkin G. Beyond a good story: from Hawthorne effect to reactivity in health professions education research. Med Educ. 2017; 51(1):31-39. 


\section{Publish your work in this journal}

The International Journal of COPD is an international, peer-reviewed journal of therapeutics and pharmacology focusing on concise rapid reporting of clinical studies and reviews in COPD. Special focus is given to the pathophysiological processes underlying the disease, intervention programs, patient focused education, and self management protocols.

This journal is indexed on PubMed Central, MedLine and CAS. The manuscript management system is completely online and includes a very quick and fair peer-review system, which is all easy to use. Visit http://www.dovepress.com/testimonials.php to read real quotes from published authors.

Submit your manuscript here: http://www.dovepress.com/international-journal-of-chronic-obstructive-pulmonary-disease-journal 